\title{
Linear and Non Linear Optical Properties of Semi Organic Succinic Added Lithium Sulphate Monohydrate Crystal
}

\author{
G. Theophil Anand*, B. Praveen Kumar \\ Department of Physics and Abraham Panampara Research Centre, Sacred Heart College (Autonomous), Tirupattur - 635 601, Tamil Nadu, India.
}

\section{ART ICLE DETAILS}

\section{Article history:}

Received 24 February 2018

Accepted 10 March 2018

Available online 14 March 2018

Keywords:

Optical Analysis

Mechanical Studies

NLO Study

\begin{abstract}
A B S T R A C T
In the present work, the effect of organic dicarboxylic acid (succinic acid) on lithium sulphate monohydrate was investigated at room temperature. The presence of succinic acid in the solution grown crystals has changed the values of lattice parameters maintaining crystal structure of the host material unaltered. The wide transparency seen in the recorded UV-vis spectrum communicate that the material is applicable to produce NLO efficiency. Mechanical resistivity to the indentation of the grown crystals was estimated through Vickers's microhardness test. Enhanced second harmonic generation efficiency was achieved. All these findings advocate that the grown crystal is suitable for NLO and optoelectronic device fabrication.
\end{abstract}

\section{Introduction}

Nonlinear optics materials are prime importance of researchers as it has applications in photonics, laser technology, optical storage and electro-optic devices. In a time of information and technology, the data processing, fast data retrieving, transmission and high storage capacity sketches the attention of researchers to search for new nonlinear optical material with exceptional optical and physical properties. Semi-organic material formation is an effective approach to obtain high NLO efficiency, optical quality, and organic based nonlinear optical crystals in which a polarizable organic molecule can be bonded ionically to an inorganic host. The hybrid material retains the high optical nonlinearities of the organic molecules and favourable physical properties of the inorganic molecules, owing to their chemical co-ordination [1]. Lithium sulphate monohydrate crystal, which is grown in different methods, has piezoelectric, pyroelectric and NLO properties [2-7]. The property of Lithium ion, showing a higher charge density in comparison to all alkali metal and its ability to combine easily with organic and inorganic complexes is used to form semiorganic crystals [8] with succinic acid. Succinic acid is a naturally occurring ligand that belongs to $\mathrm{C}_{4}$ dicarboxylic acid produced as an intermediate of the tricarboxylic acid cycle. It is an important biological and industrial applicable chemicals which elucidate monoclinic crystal system with $a=5.519 \AA, b=8.862 \AA, c=5.012 \AA$ and $\beta=91.59^{\circ}$. Succinic acid crystal belongs to centrosymmetric space group $P_{21} / c_{1}$ in paraelectric phase and it becomes non centrosymmetric space group $\mathrm{P}_{21}$ in ferroelectric phase [9-11]. When succinic acid is combined with organic compound Urea it crystallizes in monoclinic space group $\mathrm{P}_{21}$ with enhanced ferroelectric property [12]. To my best of knowledge, there is no literature available on NLO property of succinic acid as it possesses the basic possible property of noncentrosymmetric nature. The present study aims at forming semiorganic material keeping succinic acid as a guest material and lithium sulphate monohydrate as a host material. Secondly to examine whether succinic acid in combination with lithium sulphate monohydrate crystallizes in centrosymmetric/noncentrosymmetric space group. Thirdly, to explore whether the chemical coordination of succinic acid with lithium sulphate monohydrate exhibits enhanced NLO efficiency. Having gone through many articles regarding single crystal grown by slow evaporation method which has NLO properties, and especially the last three articles mentioned in the literature survey has given vigour to do a project in lithium sulphate monohydrate and succinic acid. Besides, the synthesis of lithium sulphate monohydrate oxalate gives a clear idea about the semi-organic materials for NLO applications. As well as it is obvious that lithium sulphate monohydrate (LSMH) is an inorganic material and has a NLO property and maleic acid is an organic material and when both are combined together it forms as a semi-organic material for an enhanced NLO properties. Hence, the work focuses on the preparation of pure lithium sulphate monohydrate (LSMH) and succinic added lithium sulphate monohydrate (SLSMH) and comparison of the same for NLO applications.

\section{Experimental Methods}

\subsection{Materials}

Lithium sulphate monohydrate $\left(\mathrm{Li}_{2} \mathrm{SO}_{4} \cdot \mathrm{H}_{2} \mathrm{O}\right)$ (Merck) (99\% AR grade), Succinic acid (Merck) (99\% AR grade) and de-ionized water.

\subsection{Synthesis of Pure and Succinic Added Lithium Sulphate Monohydrate \\ 2.2.1 Preparation of LSMH}

Single crystal of the pure LSMH was grown by slow evaporation method at room temperature. The calculated amount of LSMH and $20 \mathrm{~mL}$ of deionized water are dissolved well using magnetic stirrer to get a saturated solution. The saturated solution were filtered and covered with porous polythene sheet. The solution was allowed to evaporate at room temperature, after 4 weeks, the single crystal were grown and it is shown in Fig. 1.

\subsubsection{Preparation of SLSMH}

Stoichiometric quantities of two components (lithium sulphate monohydrate and succinic acid molar ratios of 1:1 were employed) were separately dissolved in double distilled water by using the magnetic stirrer. The solutions were mixed and stirred well until obtaining homogeneous solution. After that the solution was filtered using Whatman paper and covered with perforated sheet, allow it for crystallization through slow evaporation. A quality crystal was harvested in 30 days. It is shown in it Fig. 1.

\subsection{Characterization of Pure and Succinic Added LSMH}

Structural analysis were confirmed by X-Ray powder diffraction (XRD), the presence the vibrational modes were identified using Fourier transform infrared spectrometry (FT-IR), UV-Visible spectrum confirm the absorption of grown crystal. The electrical property of the grown 
crystals was done using dielectric technique. The stability of the material was found by thermo gravimetric analysis (TGA) and differential thermal analysis (DTA) technique. The mechanical strength of the material was confirmed with the help of Vickers's microhardness tester. The powder SHG technique confirms the presence second harmonic generation of the material.

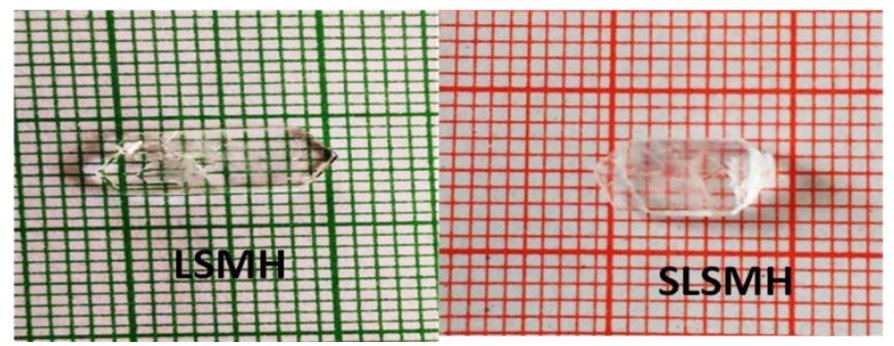

Fig. 1 Photograph of grown Crystal

\section{Results and Discussion}

\subsection{Powder X-Ray Diffraction Analysis}

The diffraction data for succinic lithium sulphate monohydrate was recorded using XPERT-PRO diffractometer with Cuk $\alpha$ radiation $(\lambda=$ $1.540598 \AA$ ). The XRD pattern of the grown crystal is shown in Fig. 2. The XRD data was analyzed with the help of the software powder X. The Bragg's reflections were indexed. The peaks intensities of the reflections $\left(\begin{array}{ll}1 & 0\end{array}\right),\left(\begin{array}{lll}0 & 1 & 1\end{array}\right),\left(\begin{array}{lll}1 & 1 & 0\end{array}\right),\left(\begin{array}{lll}0 & 1 & 2\end{array}\right)$ were increased and the additional peak observed at (2 12 ). From the observed powder XRD $2 \theta$ intensity data the crystal system confirmed to be monoclinic with space group $\mathrm{P} 2{ }_{1}$ and lattice parameters $a=15.7469 \AA, b=6.7008 \AA, c=9.4536 \AA$ angle of diffractions Alpha $=90^{\circ}$ Beta $=93.0105^{\circ}$ Gama $=90^{\circ}$, and unit cell volume $=996.15$. The increase in the intensities of the reflected planes and the appearance of the additional peak asserts the inclusion of the succinic acid into lithium Sulphate monohydrate crystals without altering the crystal structure.

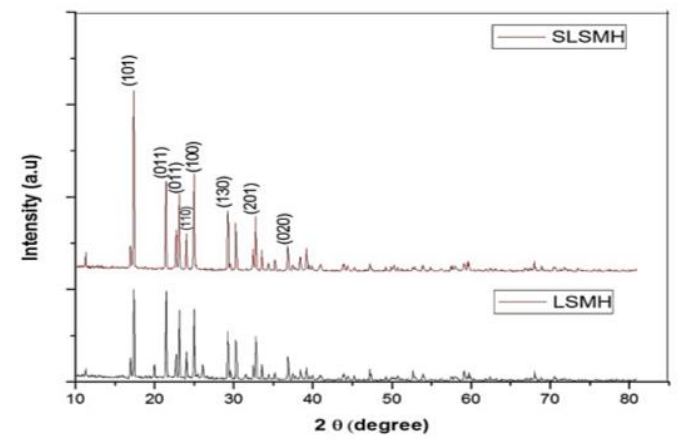

Fig. 2 Powder XRD pattern of LSMH \& SLSMH

\subsection{Optical Transmittance}

The optical transmittance spectrum was registered by employing high resolution Perkin Elmer Lambda 35 UV Visible spectrophotometer in the region $190 \mathrm{~nm}$ to $1100 \mathrm{~nm}$. The transmittance measured as a function of wavelength is portrayed in Fig. 3. The close examination of the spectrum reveals that the LSMH lower cut of wavelength is at $226 \mathrm{~nm}$ and $100 \%$ transmittance is observed in the entire visible region. The calculated optical band gap is $5.4 \mathrm{eV}$. In SLSMH lower cut of wavelength is at $240 \mathrm{~nm}$ and $100 \%$ transmittance is observed in the entire visible region. The calculated optical band gap is $5.1 \mathrm{eV}$. This would enable the higher second harmonic throughput [9] and qualify this as a favorable material to fabricate optoelectronic devices.
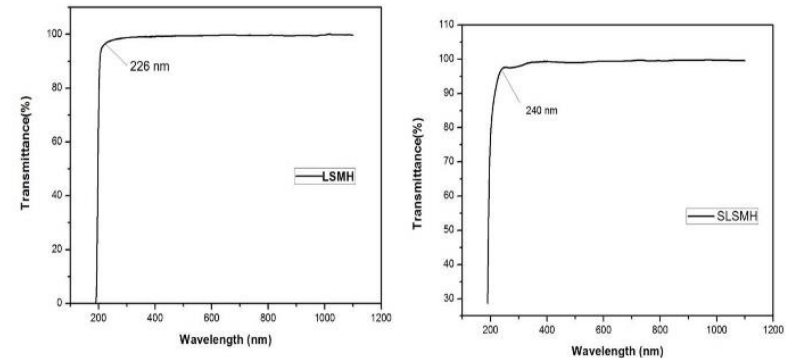

Fig. 3 UV-VIS analysis of Pure LSMH and SLSMH https://doi.org/10.30799/jnst.103.18040108

\subsection{Thermal Analysis of LSMH and SLSMH crystals}

The TGA and DTA curve of LSMH crystals were shown in Fig. 4. In The water molecules will be evaporated at the temperature of $103^{\circ} \mathrm{C}$ of LSMH. The phase transistion takes place $103^{\circ} \mathrm{C}$ up to $127^{\circ} \mathrm{C}$ from the DTA curve, there is oneexothermic and one endothermic peak was observed DTA endothermic peak observed. In TGA, it is found that SLSMH is stable upto $126^{\circ} \mathrm{C}$. The phase transition takes place $126^{\circ} \mathrm{C}$ and continuous up to 191 ${ }^{\circ} \mathrm{C}$. The melting point of LSMH and SLSMH has sharp melting point this means the crystal has no impurity.
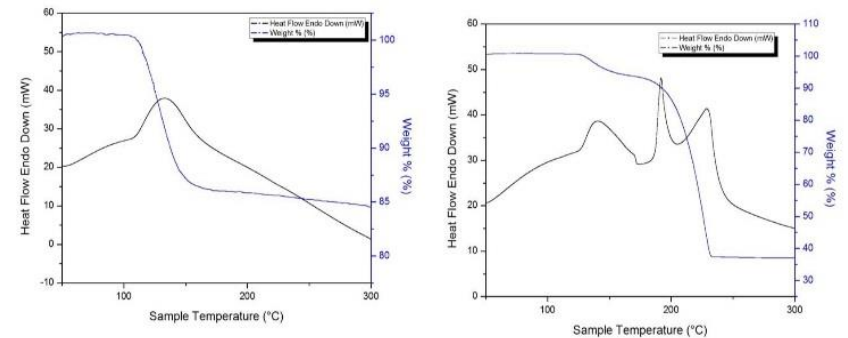

Fig. 4 Thermal studies of Pure LSMH and SLSMH

\subsection{Mechanical Hardness}

An important improvement in the semi-organic crystals over their organic counterparts is their higher mechanical hardness. The typical Vickers hardness value was measured for succinic acid lithium sulphate monohydrate under loads from 25 to $100 \mathrm{~g}$ in the selected planes. The diagonal lengths resulted due to the application of load were measured. By using the relation that relates $P$ the load in $\mathrm{kg}$ and $\mathrm{d}$ the diagonal length of the impressions in mm, the Vicker's microhardness was estimated. The formula is given by $\mathrm{Hv}=1.8544 \mathrm{P} / \mathrm{d}^{2}\left(\mathrm{~kg} / \mathrm{mm}^{2}\right)$. The perfect pattern around the mechanical indentation is illustrated in Fig. 5. There is an increase in microhardness number owing to increase in the indentation which was specified by the reverse indentation effect. This signifies the plastic deformation nature of SLSM crystals. On account of less interaction between stress and gliding plane, the dislocation propagates so easily at lower loads; but it is just reversed in case of increasing loads [13]. The work hardening coefficient of SLSM crystal was calculated using the relation $\mathrm{P}=\mathrm{adn}$. The Mayer's index (work hardening coefficient) was calculated from $\mathrm{P}=$ and $\log \mathrm{P}=\log \mathrm{a}+$ nlogd where $\mathrm{a}$ is constant for a given material. This value was attained by finding slop of the plot of log d versus $\log \mathrm{P}$. The value of $\mathrm{n}$ was gauged to be 2.77 by fitting data through least square fit method. Since the value $n=2.77$ is greater than 1.6 , the material belongs to soft material category as per the findings of Onitsch [14].

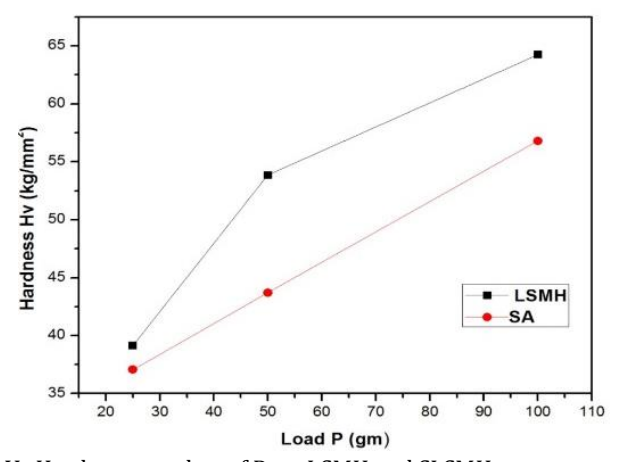

Fig. 5 Load Vs Hardness number of Pure LSMH and SLSMH

\subsection{Nonlinear Optical Property}

The second harmonic generation efficiency was estimated by powder technique of Kurtz and Perry [15]. The powder sample of succinic lithium sulphate monohydrate was irradiated with the incident radiation of wavelength1064 nm from Nd: YAG laser source. The result indicates that the powder SHG efficiency of SLSM is improved to1.03 times higher than that of KDP.

\section{Conclusion}

Crystals of succinic lithium sulphate monohydrate was grown by slow evaporation technique at ambient conditions. Crystal system and lattice parameters were confirmed by powder X-ray diffraction analysis. Change in the intensity of the peaks and additional peaks were observed. Functional groups of the grown crystal were clearly confirmed by the FTIR 
study. Slight shift in the characteristic vibrational frequency was noticed. The transparency of the crystal in the testing region was studied from UVVisible spectrum. High transparency was realized in the visible region. The mechanical strength and opto-electronic properties of the crystals were examined through Vicker's microhardness test and dielectric measurements. Increasing Vicker's hardness value with respect to load signifies less dislocation and improved quality of crystals. The enhanced NLO efficiency estimated through Kurtz and Perry powder technique enable this material suitable for NLO device applications.

\section{References}

[1] S. Brahadeeswaran, V. Venkataramanan, J.N. Sherwood, H.L. Bhat, Crystal growth and characterization of semiorganic nonlinear optical material: sodium p-nitrophenolate dehydrate, J. Mater. Chem. 8(3) (1998) 613-618.

[2] A. Silambarasan, E. Nageswara Rao, S. Venugopal Rao, P. Rajesh, P. Ramasamy, Bulk growth, crystalline perfection and optical characteristics of inversely soluble lithium sulphate monohydrate single crystals grown by conventional solvent evaporation and modified Sankaranarayanan - Ramasamy method, Cryst. Eng. Comm. 18 (2016) 2072-2080.

[3] A. Silambarasan, P. Rajesh, P. Ramasamy, Growth kinetics and bulk growth of inversely soluble lithium sulphate monohydrate single crystals and their characterization, J. Cryst. Growth 468 (2016) 38-42.

[4] A. Silambarasan, P. Rajesh, P. Ramasamy, Nucleation kinetics and growth aspects of negative solubility lithium sulphate monohydrate single crystal, J. Cryst. Growth 409 (2015) 95-99.
[5] T. Balakrishnan, K. Ramamurthi, Growth and characterization of glysine lithium sulphate single crystal, Cryst. Res. Technol. 41 (2006) 1184-1188.

[6] R. Sankar, C.M. Raghavan, R. Jayavel, Bulk growth and characterization of semiorganic nonlinear optical bis thiourea bismuth chloride single crystals, Cryst. Growth Design 7(3) (2007) 501-505.

[7] L. Bayajargal, Nonlinear optical properties of lithium sulfate monohydrate, Cryst. Res. Technol. 43 (2008) 1138-1142.

[8] H. Yadav, N. Sinha, B. Kumar, Growth and characterization of new semiorganic nonlinear optical and piezoelectric lithium sulfate monohydrate oxalate single crystals, Mater. Res. Bull. 64 (2015) 194-199.

[9] M.P. Binitha, P.P. Pradyumnan, Studies on growth, thermal, and dielectric behavior of calcium succinate trihydrate single crystals, J. Cryst. Growth 396 (2014) 38-44.

[10] B.K. Singh, N. Sinha, N. Singh, K. Kumar, M.K. Gupta, Binay Kumar, Structural, dielectric, optical and ferroelectric property of urea succinic acid crystals grown in aqueous solution containing maleic acid, Jour. Phys. Chem. Solids 71 (2010) 1774-1779.

[11] Elly Van Der Voort, The morphology of succinic acid crystals: the role of solvent interaction, J. Cryst. Growth 110 (1991) 662-668.

[12] S. Krishnan, C. Justin Raj, S. Jerome Das, Growth and characterization of novel ferroelectric urea-succinic acid single crystals, J. Cryst. Growth 310 (2008) 3313-3317.

[13] K. Ambujam, S. Selvakumar, D. Prem Anand, G. Mohamed, P. Sagayaraj, Crystal growth, optical, mechanical and electrical properties of organic NLO material $\gamma$-glycine, Cryst. Res. Technol. 41(7) (2006)671-677

[14] E.M. Onitsch, The present status of testing the hardness of materials, Mikroskopie 95 (1956) 12-14.

[15] S.K. Kurtz, T.T Perry, A second harmonic analyzer for the detection of noncentrosymmetry, Jour. Appl. Phys. 39 (1968) 145-158. 